\title{
Tétanos après application de topique traditionnel sur une dent cariée
}

\author{
Alain Kassi ${ }^{1}$, Gisèle Kouakou ${ }^{1}$, Camille Assouan ${ }^{2}$, Chrisostome Mossou ${ }^{1}$, Adama Doumbia ${ }^{1}$,Emmanuel Bissagnené \\ Service des maladies infectieuses et tropicales, CHU de Treichville, 01 BP V3, Abidjan, Côte d'Ivoire \\ Service de chirurgie maxillo-faciale et stomatologie, CHU de Treichville, 01 BP V3, Abidjan, Côte d'Ivoire \\ Correspondance : camilleass@yahoo.fr
}

(Reçu 17 juillet 2015, accepté le 31 aôut 2015)

Mots clés :

tétanos / carie dentaire / topiques traditionnels

Key words:

tetanus / dental cavity / traditional topic
Résumé - Introduction : La possibilité de survenue du tétanos après application d'un topique traditionnel pour le traitement d'une lésion carieuse dentaire est une réalité en milieu tropical. C'est ce que rapporte ce cas clinique. Observation : Une patiente de 20 ans s'était présentée avec un trismus, des contractures généralisées et des cervicalgies paroxystiques une semaine après l'application d'un topique traditionnel dentaire au niveau d'une 37 présentant une importante lésion carieuse. À son entrée à l'hôpital, elle avait une température de $38^{\circ} \mathrm{C}$, une fréquence cardiaque à $114 / \mathrm{mm}$ et une tension artérielle à 100/70 mm Hg. Aucune notion de blessure n'était retrouvée à l'interrogatoire et la couverture vaccinale antitétanique n'était pas à jour. Le diagnostic de tétanos était d'emblée évoqué. Une sérothérapie antitétanique associée à une antibiothérapie et à un traitement par benzodiazépine, des soins locaux ont aussi été prodigués. L'évolution a été favorable en 11 jours. Discussion : Ce cas de tétanos est très inhabituel. La présence de spore tétanique peut s'expliquer par la contamination de certains composants du topique appliqué dans la lésion carieuse, en particulier les feuilles de tabac et certaines plantes traditionnelles lors de leur séchage sur un sol souillé. Conclusion : Une lésion carieuse dentaire apparaît comme une authentique porte d'entrée du tétanos après application d'un topique à base de plantes ayant séjourné sur un sol souillé.

\begin{abstract}
Tetanus after application of traditional topical treatment to a decayed tooth. Introduction: Onset of tetanus after application of a dental topical treatment to a decayed tooth may arise in tropical countries of Africa. This is illustrated by the following case report. Observation: A young female patient, 20 years old, was admitted to hospital with moderate trismus, muscular stiffness and acute cervical pain one week after dental application of a topical treatment based on medicinal plants and tobacco leaves to a decayed tooth. A temperature of $38^{\circ} \mathrm{C}$, a heart rate of 114 beats $/ \mathrm{mm}$ and a blood pressure of $100 / 70 \mathrm{~mm} \mathrm{Hg}$ were recorded. No wounds were reported and the tetanus vaccination was not up to date. The diagnosis of tetanus was immediately suggested. Antitetanic serotherapy (immunoglobulin), an antibiotic (amoxicillin and clavulanic acid) and a myorelaxant (benzodiazepine) were used successfully. Local treatments were also performed. Eleven days after, a favorable outcome was recorded. Discussion: This is an unusual case of tetanus. The presence of tetanus spores could be explained by the composition of the traditional topic preparation used on a decayed tooth, based on tobacco leaves and other medicinal plants that were dried on a soiled ground. Conclusion: Dental decay and a traditional treatment based on plants that were dried on a soiled ground appeared to be a possible source of contamination for tetanus.
\end{abstract}

\section{Introduction}

Le tétanos est une toxi-infection aiguë non immunisante due à un bacille tellurique, Clostridium tetani. Il demeure encore un problème de santé publique dans les pays en développement malgré l'existence d'un programme mondiale d'immunisation [1]. La possibilité de survenue du tétanos à la faveur d'une lésion buccodentaire a déjà été rapportée [2], mais peu décrite dans la littérature [3]. Les auteurs rapportent une observation de tétanos survenu après une application d'un 
topique traditionnel fait d'un mélange de poudre de tabac à chiquer et de feuilles de plantes séchées sur une dent cariée.

\section{Observation}

Une patiente de 20 ans, était admise pour une raideur de la nuque avec un trismus. La symptomatologie avait débuté deux jours plus tôt par un trismus avec des cervicalgies. Elle avait reçu la Méphénésine (Décontracty ${ }^{\circledR} 2$ comprimés $\times 3 / \mathrm{j}$ ) sans succès. L'histoire rapportait une douleur dentaire intense sans notion de traumatisme mandibulaire, rebelle au paracétamol. Une semaine avant l'apparition du trismus, on retrouvait une notion d'application de cataplasme fait d'un mélange de poudre de tabac à chiquer et de feuilles de plantes séchées au niveau de la 37 cariée. La couverture vaccinale antitétanique n'était pas à jour. Aucune notion de blessure n'était signalée les jours précédents le début des symptômes. À l'entrée, la patiente était consciente ; la température à $36,8{ }^{\circ} \mathrm{C}$, le pouls à 114 battements $/ \mathrm{mn}$, la tension artérielle à $100 / 70 \mathrm{~mm} \mathrm{Hg}$, le poids à $58 \mathrm{~kg}$, une ouverture buccale à $2 \mathrm{~cm}$, une contracture des muscles de la face et du cou. L'examen de la cavité orale montrait la 37 cariée. On notait la présence de tartre au niveau des régions molaires et une absence de 36,45 et 46 avec une langue d'aspect normal. L'inspection cutanée montrait une peau indemne de toute blessure. La survenue de paroxysmes musculaires en cours d'examen et la positivité de la manœuvre de l'abaisse-langue captif d'Armengaud avaient permis d'évoquer le diagnostic de tétanos généralisé. Cette manœuvre consiste à chatouiller à l'aide d'un abaisselangue, le voile du palais sans faire vomir le patient. En cas de tétanos, on observe une fermeture brusque des arcades dentaires empêchant le retrait de l'abaisse-langue. Le traitement associait une sérothérapie intrathécale en vue de neutraliser la toxine circulante avec du sérum antitétanique d'origine équine $1500 \mathrm{UI}$ et $25 \mathrm{mg}$ d'hydrocortisone pour prévenir un choc anaphylactique ; une antibiothérapie parentérale avec l'amoxicilline-acide clavulanique $(1 \mathrm{~g} / 8 \mathrm{~h})$. En outre, elle avait reçu en alternance $20 \mathrm{mg}$ de diazépam dans $500 \mathrm{~mL}$ de sérum salé isotonique en trois perfusions par jour, un litre par jour de sérum glucosé à $10 \%$ associé à un litre de ringer lactate. Les soins locaux consistaient en l'ablation des corps étrangers et le toilettage de la cavité orale avec une compresse imbibée de chlorhéxidine. Une première dose de vaccin antitétanique était administrée en cours hospitalisation. L'hémogramme montrait une leucocytose à $5200 / \mathrm{mm}^{3}$ avec $84 \%$ de neutrophiles, les plaquettes à $196000 / \mathrm{mm}^{3}$, et le taux d'hémoglobine à $11,2 \mathrm{~g} / \mathrm{dL}$. L'examen cytobactériologique du liquide céphalo-rachidien était normal. Après 11 jours de traitement, on notait une normalisation de l'ouverture buccale et une disparition des paroxysmes musculaires (Fig. 1). La radiographie panoramique dentaire montrait les dents 16 et 37 cariées avec la présence d'une parodontite apicale aiguë au niveau de la 37 (Fig. 2). Il n'existait pas de lésions osseuses mandibulaires et l'intégrité de l'articulation temporo-mandibulaire était conservée. La sortie était alors autorisée et

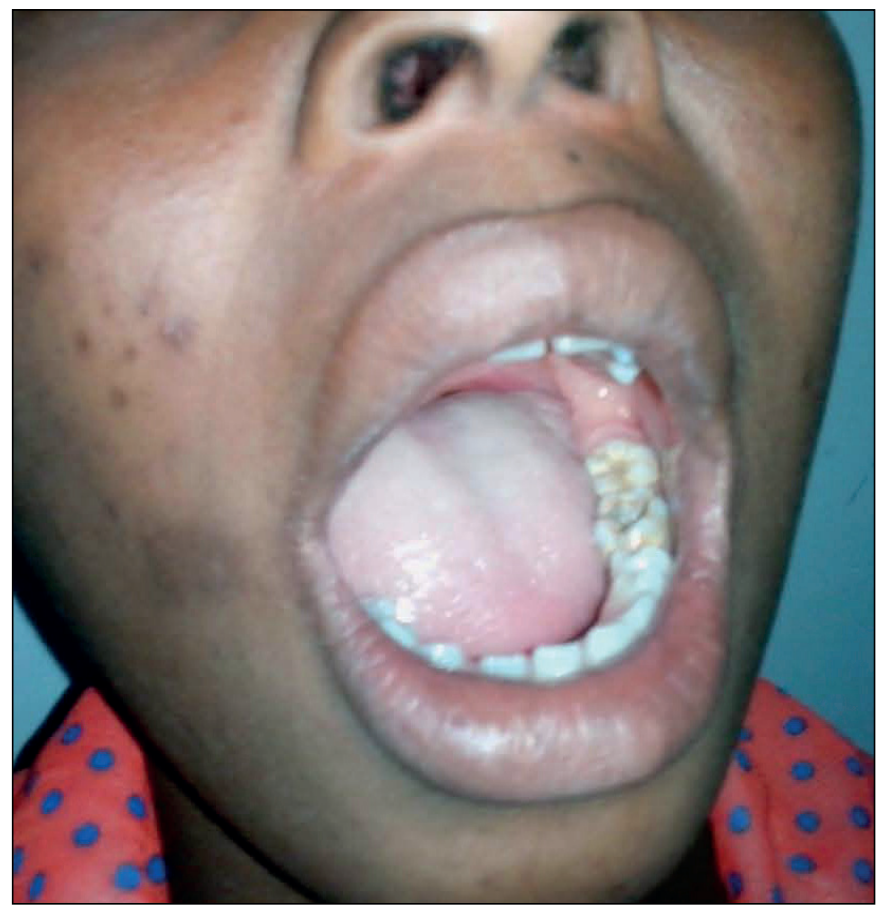

Fig. 1. 11 jours après traitement, le trismus est levé. L'importante lésion carieuse au niveau de 37 est bien visible.

Fig. 1. 11 days after treatment, trismus was over. The important decay on 37 is well discernible.

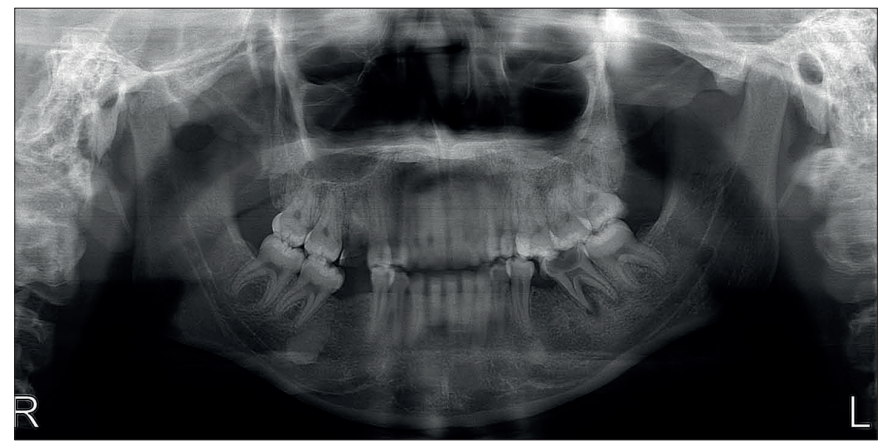

Fig. 2. Radio panoramique montrant les dents 16 et 37 cariées avec la présence d'une parodontite apicale aiguë au niveau de 37 .

Fig. 2. Panoramic X-ray showing teeth 16 and 37 decayed with an acute apical periodontitis at the apex of 37 .

une deuxième dose de vaccin antitétanique a été administrée trente jours après la première dose.

\section{Discussion}

Cette observation rapporte un cas authentique de tétanos survenu au décours d'une application de topique traditionnel fait d'un mélange d'eau, de poudre de tabac à chiquer et de feuilles de plantes séchées au niveau d'une dent cariée. 


\section{CAS C L I N I Q U E}

Par définition, le tétanos est dû à un germe tellurique et sa survenue nécessite la présence de spore tétanique au niveau d'une porte d'entrée avec des conditions d'anaérobiose. Dans cette observation, les modalités de contamination de 37 et 16 par la spore tétanique peuvent être discutées. En effet, la présence de la spore tétanique au niveau dentaire peut s'expliquer par la contamination des feuilles de tabac et des plantes traditionnelles lors du séchage où elles sont le plus souvent en contact avec les sols souillés par les spores qui peuvent y survivre pendant de nombreuses années et résister à la plupart des désinfectants [4]. Le tabac est bien connu pour ses propriétés antalgiques liées à la nicotine. Il est traditionnellement utilisé par certains peuples pour soulager des douleurs [5]. L'obstruction de la cavité carieuse par le topique traditionnel a probablement favorisé les conditions d'anaérobiose propice à la croissance du germe et à la libération de la toxine responsable de la survenue du tétanos.

Cette observation présente deux particularités :

- d'une part le sexe de la patiente ; en effet dans la littérature, le tétanos est plus fréquent chez les hommes du fait de certaines professions à risque [6]. Chez la femme, le tétanos est moins fréquent à cause de l'existence d'un programme d'immunisation antitétanique gratuit pour les femmes en âge de procréer afin de les protéger et éviter le tétanos néonatal [1] ;

- d'autre part, la porte d'entrée dentaire; elle constitue une originalité puisqu'elle est rare. C'est la précision par l'interrogatoire de la notion d'application de topique traditionnel dans la cavité carieuse qui a permis de faire le lien avec la symptomatologie.

L'évolution chez la patiente a été favorable à l'instar des cas rapportés par certains auteurs [2,3].

\section{Conclusion}

Cette observation montre que le tétanos à porte d'entrée dentaire est une réalité en milieu tropical. Elle souligne la nécessité de sensibiliser les populations aux risques liés à certaines pratiques traditionnelles et rappelle l'intérêt du respect du schéma vaccinal antitétanique.

\section{Conflits d'intérêt : aucun}

\section{Références}

1. WHO/AFRO - Progress towards maternal and neonatal elimination (MNTE) in the African Region. Vaccine Preventable Diseases Bulletin 2003;36:1-3.

2. St-Hilaire $H$, William $D$, Weber, Ramer $M$, Lumerman $H$. Clinicopathologic conference: trismus following dental treatment. Oral Surg Oral Med Oral Pathol Oral Radiol Endod 2004;98:261-266.

3. Anzouan-Kacou E, Assouan C, Aba T, Nguessan D, Adouko J, Salami A, et al. Tetanus post avulsion dentaire. À propos d'une observation. Odonto Stomatol Trop 2015;38:10-12.

4. Ryan J, R. Clostridium, Peptostreptococcus, Bacteroids, and other Anaerobes. In: Ryan KJ, Ray CG Eds. Sherris Medical Microbiology: An Introduction to Infectious Diseases. 4th ed. USA: McGraw-Hill, 2004:309-326.

5. Bruneton J. Pharmacognosie - Phytochimie, plantes médicinales, 4e éd., revue et augmentée, Paris: Tec \& Doc - Éditions médicales internationales, 2009.

6. Aba T, Kra 0, Ehui E, Tanon KA, Kacou AR, Ouatara B, et al. Aspects cliniques et évolutifs du tétanos lié aux soins dans le service de référence du CHU d'Abidjan. Bull Soc Pathol Exot 2011;104:38-41. 